\title{
Comparación pruebas de campo para determinar mastitis subclínica en bovinos, Pomacochas, región Amazonas, Perú
}

\section{Comparison of field tests to determine subclinical mastitis in cattle, Pomacochas, Amazonas region, Peru}

\author{
Carlos Ulises Escobedo Jalk ${ }^{1}$
}

\section{RESUMEN}

La investigación tuvo por objetivo comprobar dos pruebas de campo para determinar Mastitis Subclínica en bovinos, en la Localidad de Florida-Pomacochas, 2019. Para el desarrollo de la investigación se utilizó el método biológico (Prueba California Mastitis Test) y el método químico (Prueba de Whiteside) los cuales permitieron comprobar cuál de las pruebas son más eficientes para detectar la mastitis bovina. Se encontró un $47 \%$ de efectividad en la prueba Whitside y el 53\% en la prueba California Mastitis Test, lo que dificulta la confiabilidad por parte de los criadores de ganado con ambas pruebas; pero si se tiene que utilizar una de las dos la más recomendables es la California Mastitis Test. Se concluye que la prevalencia de mastitis subclínica está representada por el $27 \%$ del total de bovinos evaluados.

Palabras clave: California mastitis test, prevalencia mastitis

\begin{abstract}
The objective of the research was to verify two field tests to determine Subclinical Mastitis in bovines in the town of Florida-Pomacochas, 2019. For the development of the research, the biological method (California Mastitis Test) and the chemical method (Whiteside Test) were used, which allowed us to verify which of the tests are more efficient to detect mastitis bonina. $47 \%$ effectiveness was found in the Whitside test and $53 \%$ in the California Mastitis Test, which hinders the reliability of cattle breeders with both tests; But if you have to use one of the two, the most recommended is the California Mastitis Test. It is concluded that the prevalence of subclinical mastitis is represented by $27 \%$ of the total number of cattle evaluated.
\end{abstract}

Keywords: California mastitis test, prevalence mastitis

${ }^{1}$ Bachiller en Ingeniería Zootecnista. Universidad Nacional Toribio Rodríguez de Mendoza de Amazonas. Correo Electrónico: carlosjalk_02@hotmail.com 


\section{INTRODUCCIÓN}

Hoy en día la propagación de microorganismos en diferente índole han sido la causa de infecciones intramamarias en las diversas regiones del mundo, hecho que ha permitido estudios diversos mediante los cuales se ha establecido una división de patógenos en ambientales y contagiosos; todo esto teniendo en cuenta además la interrelación de la epidemia con el grado causante de contagio.

Un factor preponderante son las unidades de producción entorno a la rentabilidad de los productores, sin embargo, las distorsiones y perturbaciones en la fertilidad conjuntamente con la mastitis son una de los principios que afecta al crecimiento económico de los criadores de ganado en todas las regiones y partes del planeta. Ambos factores ocasionan pérdidas importantes en la producción y la calidad del producto y con ello disminuye el precio e aumenta los costos de inversión para combatirlo.

Según Zendejas (2020) el incremento permanente de células anatómicas en los infectados por de mastitis generan distorsiones en las composiciones químicas de la leche siendo más frecuente la minimización en calcio, fosforo, grasas y proteínas; causando incrementos secuenciales en la acumulación de fermento en leche. Mediante este se comprimen importantes dosis de lactosa y proteína, todo esto conjuntamente de constantes alteraciones en la caseína por factores operacionales de enzimas proteolíticas y microorganismos; los mismos que producen bacterias y leucocitos afectando al bajo rendimiento y la disminución del periodo en la vida útil del producto.

Según López (2017) para el Consejo Nacional de Estados Unidos existe un incremento en las inversiones de los productores los últimos años, todo esto a partir de que gran parte de las vacas son afectadas por mastitis siendo un valor representativo un tercio de la población. Así mismo Canadá y Cuba están en las regiones con más perdidas siendo 200 dólares por cada vaca a año, valores que corroboran lo mencionado líneas atrás en donde el 70 u $80 \%$ del total de pérdidas tienen relación con la mastitis subclínica, así como en un 20 a 30\% a la denominada mastitis clínica.

Perú no ha sido ajeno a las pérdidas económicas por causa de la mastitis (clínica y subclínica), pues según Velásquez (2017) con su investigación realizada en Huacho San Felipe refirió que el $48 \%$ de los individuos que formaron parte de la población cuentan con mastitis subclínica con prevalencia de células anatómicas. Siendo participe de la investigación 4364 vacas Holstein dividido en 32 establos.

\section{MATERIAL Y MÉTODO}

La muestra fue establecida teniendo en cuenta el muestreo aleatorio simple para poblaciones finitas siendo $\mathrm{Z}=95 \%$ y e $=10 \%$, así como $\mathrm{P}=50 \%$ y $\mathrm{Q}=$ $50 \%$ así mismo para tener una mejor precisión y mejor representación de la población se realizó la fijación proporcional de bovinos, para finalmente ser distribuidos de manera estratificada según sector.

M1= Aplicando la formula se tomó la siguiente distribución muestral según Raza y por sectores determinados para el estudio.

- Método Biológico (Prueba California Mastitis Test)

La prueba de CTM se realizó según el horario de ordeño según sea el caso en la mañana o en la tarde, en vacas desde las dos semanas después del parto hasta los 5 meses de producción, tomando una cantidad de $2 \mathrm{ml}$ de cada uno de los integrantes de la muestra. Para la recolección de la información se tuvo en cuenta una ficha técnica por bovino que nos permitió describir e identificar casos negativos y/o positivos según edad, época de lactancia, números de partos, entre otros.

Para la prueba California Mastitis Test se mescló el reactivo con una cierta cantidad de leche. Es así que este reactivo estuvo compuesto por alquilarilsulfonato que refiere a un desinfectante aniónico, el mismo que diluye la membrana celular y nuclear con la invariable expulsión de ADN quien al mezclarse con la desinfectante forma una especie de gel. Con ello cuanto más ADN se muestre aumentará la densidad del gel, y con ello resulta preciso indicar la existencia de gran cantidad de células anatómicas en la leche librando ese componente (Nacional Mastitis Council, 1996).

\section{- Método Químico (Prueba de Whiteside)}

Una vez hecho el trabajo de campo con CMT, se procedió a realizar la prueba química en vacas desde las dos semanas después del parto hasta los 5 meses de producción, donde se mezcló la leche con hidróxido de sodio en un $4 \%$, este origina que la leche congele en pequeñas porciones que ya puede ser observado a primera vista. Las porciones aumentaran en tamaño dependiendo de la cantidad de células anatómicas que este contenga. Así también para ser un tanto más perceptible es preciso usar placa acrílica color negro que en la investigación tuvo 4 separadores. 


\section{RESULTADOS}

\section{Figura 1}

Raza de los bovinos en el sector San Lorenzo

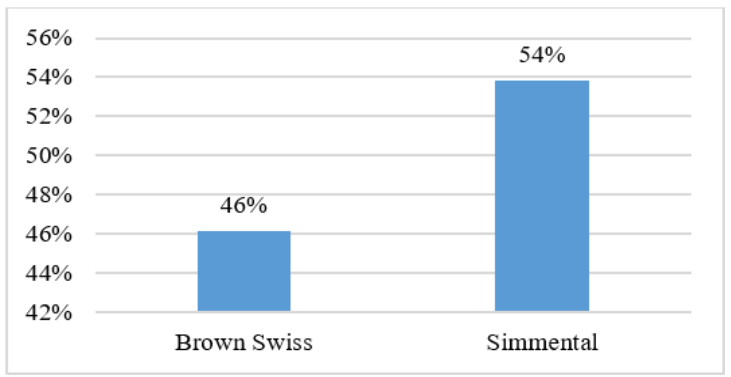

En la figura 1, se muestra que el simmental es el bovino que más se cría en el sector de San LorenzoFlorida con el $54 \%$ y con el $46 \%$ crían raza Brown Swiss.

\section{Figura 2}

Raza de los bovinos en el sector Miraflores de Levanto.

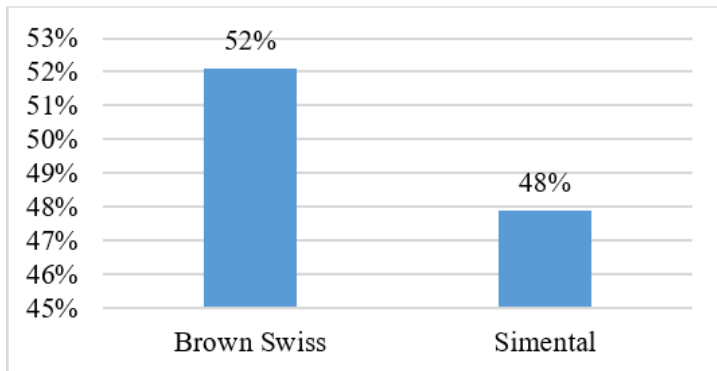

En la figura 2, se muestra que según la variable raza en el sector Miraflores de Levanto la raza que predomina es la raza Brown Swiss con el 52\% y solo el $48 \%$ son de raza Simmental.

\section{Figura 3}

Distribución según el número de partos en la Localidad de Florida-Pomacochas según los sectores: San Lorenzo y Miraflores de Levanto.

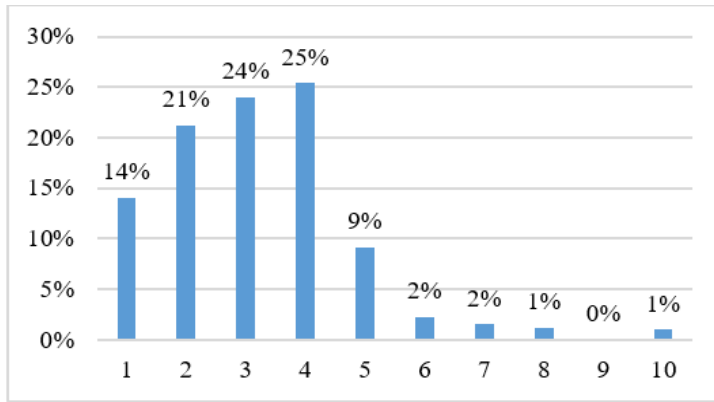

En la figura 3, se muestra que el $25 \%$ de los bovinos evaluados se tuvo vacas con 4 partos en mayor medida, seguido por el $24 \%$ con 3 partos; y por último en menor porcentaje los bovinos tuvieron 8 y 10 partos con el $1 \%$.

\section{Figura 4}

Prevalencia de mastitis subclínica por cuartos afacetados según la Prueba California Mastitis Testy la Prueba de Whiteside

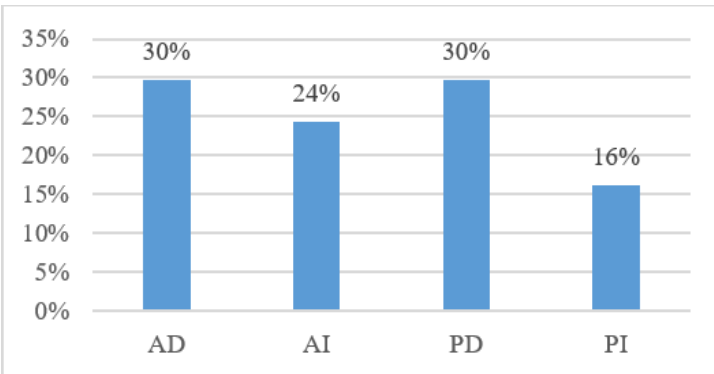

En la figura 4, se muestra que en mayor medida con el $30 \%$ fueron afectados el Anterior Derecho y el Posterior Derecho Respectivamente; así mismo con el $24 \%$ fue afectado el anterior izquierdo y por último en posterior izquierdo con un $16 \%$.

\section{Figura 5}

Prevalencia de mastitis subclínica de acuerdo al número de partos según la Prueba California Mastitis Test, sector Miraflores de Levanto.

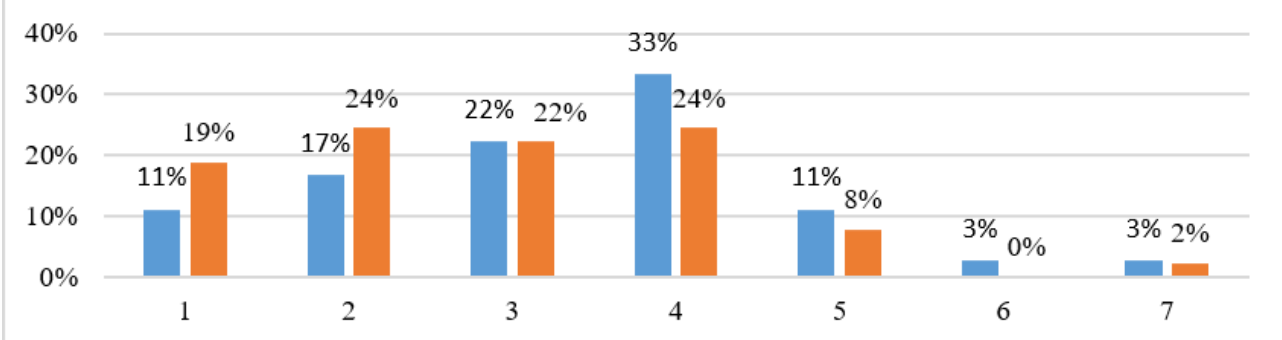


En la figura 5, se observa que se encontró de manera positivo con $33 \%$ en los bovinos que tuvieron 4 partos, y negativo en mayor medida también en las vacas de 4 partos y de 2 partos con un $24 \%$ de manera equitativa.

\section{Figura 6}

Prevalencia de mastitis subclínica de acuerdo al número de partos según la Prueba California Mastitis Test, sector San Lorenzo.

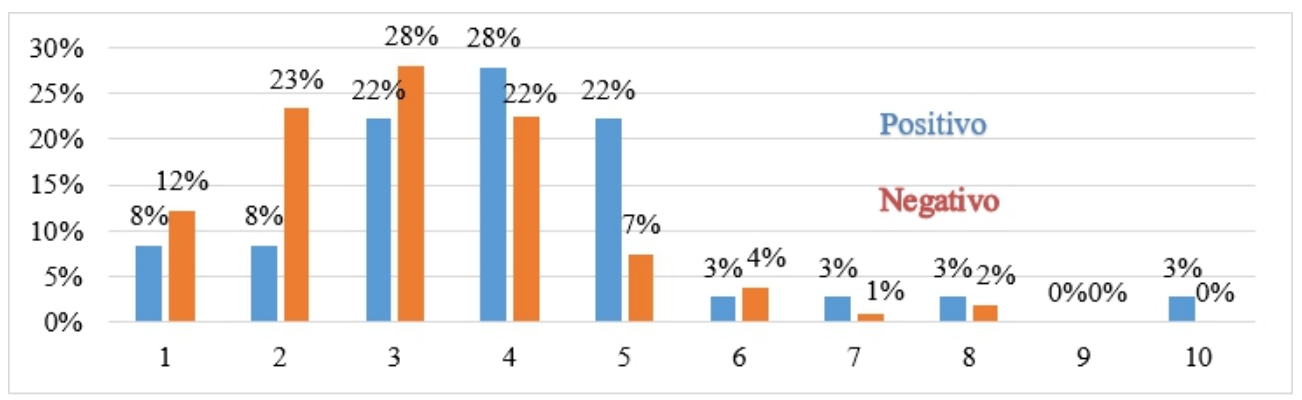

En la figura 6 , se muestra que un $28 \%$ se evidencio que las vacas de 4 partos tuvieron más dando positivo, y de manera negativa con un $28 \%$ fueron las vacas de 3 partos.

\section{Figura 7}

Prevalencia de mastitis subclínica de acuerdo al número de partos según la Prueba Whiteside, sector Miraflores.

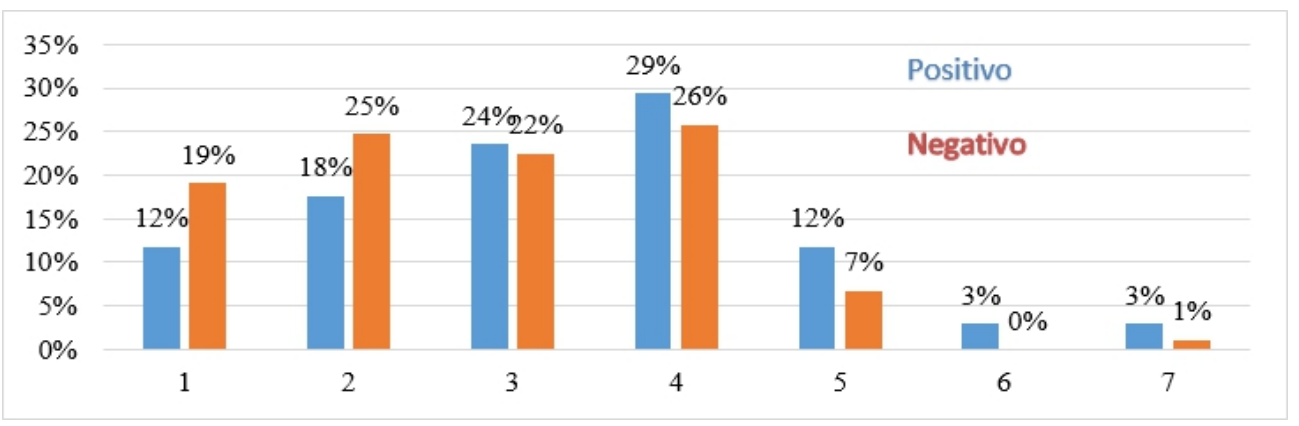

En la figura 7, se muestra que en mayor medida con el $29 \%$ hacen referencia a la positividad generalmente en bovinos de 4 partos; así también en las vacas de 4 partos se evidenció en mayor medida el lado negativo de la prevalencia de mastitis subclínica con un $26 \%$.

\section{Figura 8}

Prevalencia de mastitis subclínica de acuerdo al número de partos según la Prueba Whiteside, sector San Lorenzo.

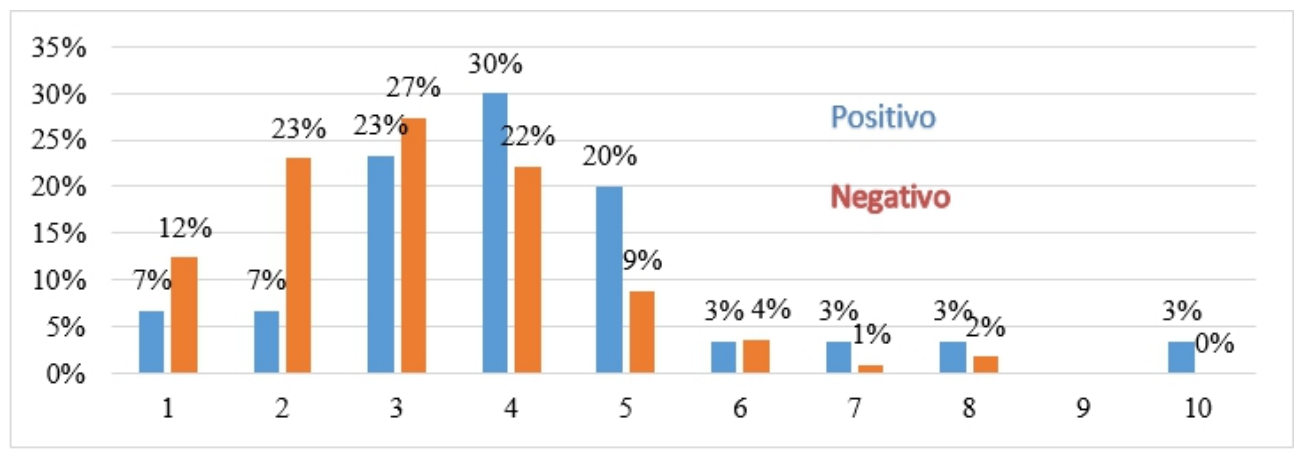

En la figura 8, se evidenció que las vacas de 4 partos con un 30\% en mayor medida dieron positivo a mastitis sub clínica, y $27 \%$ las vacas de 3 dieron negativo 


\section{Figura 9}

Grados de infección total según el número de partos y por cuartos afectado, teniendo en cuenta la prueba California Mastitis Test y la prueba Whiteside.

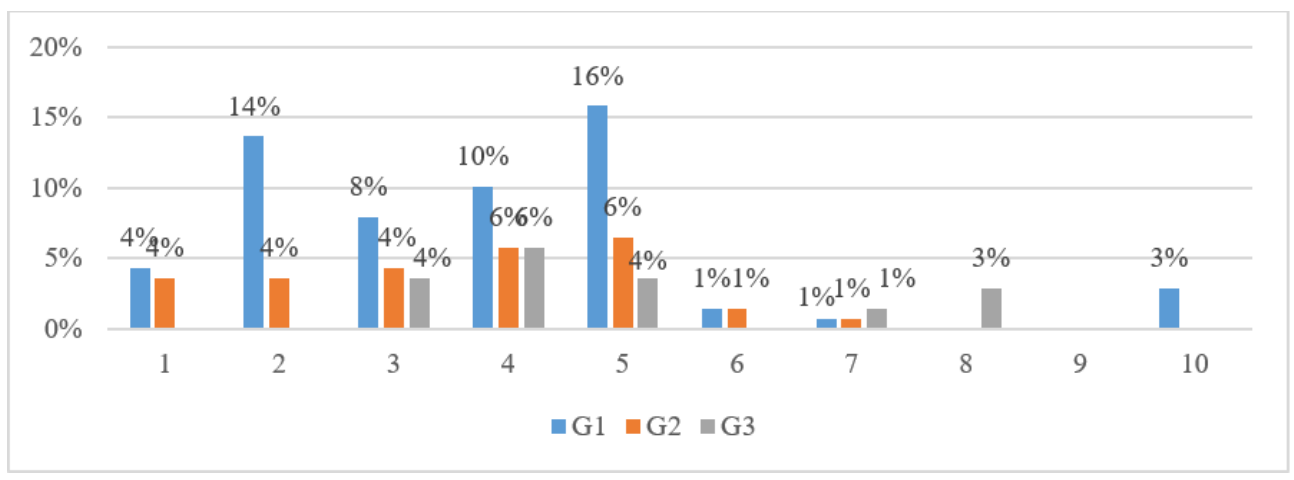

En la figura 9, se muestra el grado de infección que más se representa es el de grado 1 con 16\% siendo los bovinos de 5 partos los que más fueron afectados.

\section{Figura 10}

Grados de infección total según la prueba California Mastitis Test y la prueba Whiteside.

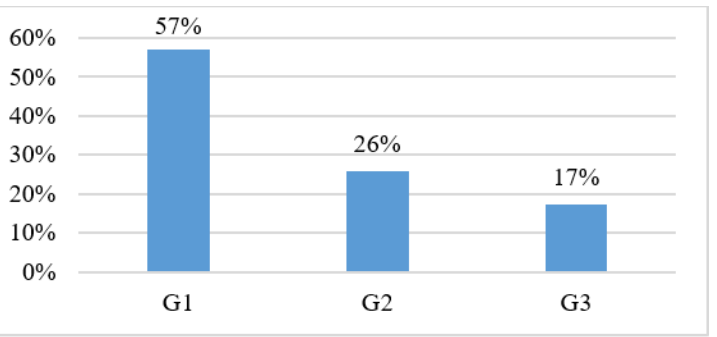

En la figura 10, se muestra que según la evaluación total respecto a los dos tipos de pruebas California Mastitis Test y la prueba Whiteside; del 100\% que dieron positivos el $57 \%$ fueron de grado 1 , el $26 \%$ de grado 2 y solo el $17 \%$ de grado 3 .

\section{Eficiencia de la prueba Whiteside y California Mastitis Test}

Para confirmar la efectividad de la prueba Whiteside se tuvo en cuenta la siguiente formula:

$\%$ de efectividad $=\frac{N^{\circ} \text { de vacas positivas según prueba Whiteside }}{\text { Total, de vacas evaluadas con mastitis }} \times 100=\frac{64}{136} \times 100=\mathbf{4 7} \%$

Teniendo en cuenta la fórmula planteada se evidencia un $47 \%$ de efectividad en la prueba Whiteside, lo que dificulta la confiabilidad por parte de los criadores de ganado.

Para confirmar la efectividad de la prueba CMT se tuvo en cuenta la siguiente formula:

$\%$ de efectividad $=\frac{\mathrm{N}^{\circ} \text { de vacas positivas según prueba CMT }}{\text { Total, de vacas evaluadas con mastitis }} \times 100=\frac{72}{136} \times 100=\mathbf{5 3} \%$

Teniendo en cuenta la fórmula planteada se evidencia un $53 \%$ de efectividad en la prueba California Mastitis Test.

\section{DISCUSIÓN}

Teniendo en cuenta la investigación de Almeida (2015), acerca de la prevalencia de mastitis bovina mediante la prueba de california mastitis test e identificación del agente etiológico, en el centro de acopio de leche en San Pablo Urco-Ecuador. Resultó que la Prevalencia de Mastitis Bovina; se fundamenta en un $45 \%$ el área de estudio, el mismo que presentó un alto grado de infección en las vacas lactantes. En ese contexto teniendo en cuenta la presente investigación existe una diferencia significativa frente a la prevalencia de mastitis, ya que del $100 \%$ de las vacas que formaron parte de la población el $27 \%$ cuenta con mastitis subclínica. Así mismo frente al estudio el $54.5 \%$ de la población analizada resultó negativo y el $45.5 \%$ tienen algún grado de mastitis con la prueba CMT; frente a este resultado existe una diferencia significativa con la investigación en la localidad de Pomacochas en los sectores de San Lorenzo y Miraflores, ya que la prevalencia de mastitis subclínica arrojó un $27 \%$ del total de bovinos evaluados. Es decir, del $100 \%$ de las vacas que formaron parte de la población el $27 \%$ cuenta con mastitis subclínica de grado 1,2 y 3 respectivamente.

La investigación desarrollada por Chamba (2019), acerca de la prevalencia de mastitis subclínica en bovinos de la asociación de ganaderos de Pueblo Nuevo de Colán- Piura - Perú, encontró que la enfermedad está estrechamente relacionadas a los factores de riesgos evaluados, pues existió más casos positivos en las vacas que tuvieron 4 a 5 años principalmente los que tuvieron por lo menos 2 partos; así mismo encontró que la parte más afectada ha sido el cuarto mamario anterior izquierdo teniendo en cuenta de manera individual y los posteriores de manera colectiva. Teniendo en cuenta el mencionado estudio la presente investigación encontró resultados distintos pues la prevalencia de mastitis subclínica 
por cuartos afacetados y por número de partos según la Prueba California Mastitis Test y la Prueba de Whiteside, en mayor medida el $30 \%$ fueron afectados el Anterior Derecho y el Posterior Derecho respectivamente; así mismo con el $24 \%$ fue afectado el anterior izquierdo y por último en posterior izquierdo con un $16 \%$.

\section{CONCLUSIONES}

La prevalencia de mastitis subclínica está representada por el $27 \%$ del total de bovinos evaluados. Es decir, del $100 \%$ de las vacas que formaron parte de la población el 27\% cuenta con mastitis subclínica. Así mismo según la prevalencia de mastitis subclínica por cuartos afectados y por número de partos según la Prueba California Mastitis Test y la Prueba de Whiteside, en mayor medida el $30 \%$ fueron afectados el Anterior Derecho y el Posterior Derecho Respectivamente; así mismo con el $24 \%$ fue afectado el anterior izquierdo y por último en el posterior izquierdo con un $16 \%$.

Teniendo en cuenta los grados de infección según la evaluación con los dos tipos de pruebas California Mastitis Test y la prueba Whiteside; del 100\% que dieron positivos el $57 \%$ fueron de grado 1 , el $26 \%$ de grado 2 y solo el $17 \%$ de grado 3 .

Se evidenció un $47 \%$ de efectividad en la prueba Whiteside y el 53\% en la prueba California Mastitis Test.

\section{REFERENCIAS BIBLIOGRÁFICAS}

Almeida, D. (2015). Prevalencia de mastitis bovina mediante la prueba de california mastitis test e identificación del agente etiológico, en el centro de acopio de leche en la Comunidad San Pablo Urco, Olmedo - Cayambe Ecuador, 2014. Quito: Universidad Politecnica Salesiana. Recuperado el 14 de Noviembre de 2019, de file://C:/Users/User/ Downloads/YT00246_unlocked.pdf

Chamba, D. (2019). Prevalencia de mastitis subclínica en vacas de la asociación de ganaderos de Pueblo Nuevo de Colán provincia de Paita - Piura - Perú 2018. (F. d. Zootecnia, Ed.) Piura: Universidad Nacional de Piura. Recuperado el 14 de Noviembre de 2019, de http://repositorio.unp.edu.pe/ bitstream/handle/UNP/1802/ZOO-CHAINF-2019.pdf? sequence $=1 \&$ isAllowed $=\mathrm{y}$

López, J. e. (2017). Levante de novillas de razas lecheras en confinamiento. Revista
Universidad de La Salle, 153-160.

Velasquez, C. (2010). Factores que influyen en la presentación de mastitis subclínica. Revista Infinitum, 16-21.

Zendejas, E. e. (29 de Mayo de 2020). Mastitis, problema número 1 en ganado lechero: Manejo, Tratamiento y Control. Bio Zoo S.A de C.V., 1-5. Obtenido de https://www.ganade ria.com/destacado/Mastitis\%2C-problemanumero-1-en-ganado-lechero $\% 3 \mathrm{~A}-\mathrm{Manejo} \%$ 2C-Tratamiento-y-Control\#: : text $=\mathrm{La} \% 20$ mastitis $\% 20$ bovina $\% 2 \mathrm{C} \% 20$ junto $\% 20$ con,la $\% 201$ eche $\% 20 \mathrm{y} \% 20 \mathrm{en} \% 20$ consecuencia 